\title{
Impact of comorbidities on postoperative complications in patients undergoing laparoscopy- assisted gastrectomy for gastric cancer
}

\author{
Mikito Inokuchi $^{{ }^{*}}$, Keiji Kato ${ }^{1}$, Hirofumi Sugita ${ }^{1}$, Sho Otsuki ${ }^{1}$ and Kazuyuki Kojima ${ }^{2}$
}

\begin{abstract}
Background: Comorbidity is a predictor of postoperative complications (PCs) in gastrectomy. However, it remains unclear which comorbidities are predictors of PCs in patients who undergo laparoscopy-assisted gastrectomy (LAG). Clinically, insufficient lymphadenectomy (LND) is sometimes performed in high-risk patients, although the impact on PCs and outcomes remains unclear.
\end{abstract}

Methods: We retrospectively studied 529 patients with gastric cancer (GC) who underwent LAG. PCs were defined as grade 2 or higher events according to the Clavien-Dindo classification. We evaluated various comorbidities as risk factors for PCs and examined the impact of insufficient LND on PCs in patients with risky comorbidities.

Result: A total of 87 (16.4\%) patients had PCs. There was no PC-related death. On univariate analysis, heart disease, central nervous system (CNS) disease, liver disease, renal dysfunction, and restrictive pulmonary dysfunction were significantly associated with PCs. Both liver disease and heart disease were significant independent risk factors for $P C s$ on multivariate analysis (odds ratio $[O R]=3.25, p=0.022 ; O R=2.36, p=0.017$, respectively). In patients with one or more risky comorbidity, insufficient LND did not significantly decrease PCs $(p=0.42)$ or shorten GC-specific survival $(p=0.25)$.

Conclusion: In patients who undergo LAG for GC, the presence of heart disease or liver disease is an independent risk factor for PC. Insufficient LND (for example, D1+ for advanced GC) might be permissible in high-risk patients, because although it did not reduce PCs, it had no negative impact on GC-specific survival.

\section{Background}

Gastric cancer $(\mathrm{GC})$ is the fourth most common malignancy [1]. At present, the worldwide treatment of choice for GC is complete surgical removal of the tumor and adjacent lymph nodes. Surgical outcomes are influenced by various factors, including patients' characteristics and concurrent disease, type of operation, and quality of care. Postoperative complications (PCs) negatively affect the quality of life of patients who undergo gastrectomy and can even be life-threatening. Identification of risk factors for PCs might help to reduce such complications, and many studies have attempted to evaluate risk factors for PCs associated with various procedures. Comorbidity has been reported to be a predictor of PCs in patients

\footnotetext{
* Correspondence: m-inokuchi.srg2@tmd.ac.jp

'Department of Surgical Oncology, Tokyo Medical and Dental University,

Tokyo, Japan

Full list of author information is available at the end of the article
}

who receive gastrectomy for GC [2-5]. However, what types of comorbidities are associated with the highest risk of PCs in patients who undergo gastrectomy remains to be fully defined. Risk factors probably differ between abdominal (surgical) and non-abdominal (medical) PCs. The primary objective of study was to clarify comorbidities associated with PCs in laparoscopy-assisted gastrectomy (LAG), a procedure for less invasive surgery increasingly used throughout the world. Clarifying specific comorbidities might contribute to improved treatment strategies for GC.

Scoring systems such as the Estimation of Physiologic Ability and Surgical Stress (E-PASS) score and the Physiologic and Operative Severity Score for the enUmeration of Mortality and morbidity (POSSUM) are useful for predicting the risks of mortality and morbidity after various operations [6,7], although they are not commonly used in clinical practice. In patients with comorbidities 
likely to adversely affect postoperative outcomes, standardized treatments, such as gastrectomy with D2 lymphadenectomy (LND) for advanced GC, tend to be avoided by surgeons. However, criteria for the selection of patients who should undergo insufficient LND and the impacts of insufficient LND on PCs and survival in high-risk patients remain to be defined. The secondary objective of this study was to evaluate the outcomes of high-risk patients who underwent insufficient LND. We verified whether insufficient LND negatively affects postoperative survival in this retrospective study.

\section{Methods}

We retrospectively identified 529 consecutive patients who underwent LAG with LND for pathological stage I to III GC in our hospital between 2003 and 2012. Patients who underwent thoracolaparotomy, emergency surgery, incomplete tumor resection, and combined operations for other malignancies were excluded. The present study was in compliance with the Declaration of Helsinki, and was approved by the ethics committee of Tokyo Medical and Dental University. In principle, early-stage GC was treated by LAG in accordance with the treatment guidelines of the Japanese Gastric Cancer Association [8]. The extent of LND was retrospectively classified as $\mathrm{D} 1, \mathrm{D} 1+(\alpha$ or $\beta)$, or D2 in accordance with the treatment guidelines, version 2 [8]. However, reduced LND was performed in patients with severe comorbidities. In patients who underwent LAG, carbon dioxide pneumoperitoneum was maintained at $10 \mathrm{~mm} \mathrm{Hg}$, and a 4- to $5-\mathrm{cm}$ incision was made in the upper abdomen or navel to remove tissue specimens and conduct anastomosis. For lymph node dissection, we used harmonic scissors and monopolar and bipolar electric cautery devices. All patients received systemic antibiotics (a first-generation cephem) several times on the day of surgery. The nasogastric tube was left in place until postoperative day 1 according to our protocol.

All patients preoperatively underwent venous blood analysis (including hemoglobin, serum albumin, and creatinine), electrocardiography, chest radiography, and pulmonary function testing, including vital capacity (VC), forced expiratory volume in 1 second (FEV1), and forced vital capacity (FVC). The results of these examinations were retrieved from the patients' electronic medical records. The following variables were obtained from our prospective GC database: patient age and gender; body mass index (BMI); comorbidities; regular use of steroids; tumor characteristics; extent of lymph-node dissection; operation time; estimated blood loss; and PCs. All comorbidities other than pulmonary and renal dysfunction were defined as conditions that required treatment. For example, heart disease included ischemic disease treated by interventional procedures, atrial fibrillation requiring anticoagulant treatment, and congenital cardiac failure treated by medication. Liver disease included both cirrhosis and chronic hepatitis treated by medication. Pulmonary dysfunction was classified into two categories on basis of the results of preoperative spirometry. Restrictive pulmonary dysfunction was defined as a predicted VC of less than $80 \%$, and chronic obstructive pulmonary disease (COPD) was defined as an FEV1/FVC ratio of less than 0.70. Renal dysfunction was defined as a serum creatinine concentration higher than the upper limit of normal according to our hospital's criteria $(>1.1 \mathrm{mg} / \mathrm{dL}$ in males and $>0.8 \mathrm{mg} / \mathrm{dL}$ in females). Anemia was defined according to the World Health Organization (WHO) criteria $(<13 \mathrm{~g} / \mathrm{dL}$ in males and $<12 \mathrm{~g} / \mathrm{dL}$ in females). Hypoalbuminemia was defined as a serum albumin concentration of less than $3.5 \mathrm{~g} / \mathrm{dL}$. In addition, some comorbidities were classified into two groups according to severity.

All patients were followed up until June 2013. The median follow-up was 52 months (5.5-126). A total of 59 (11.1\%) patients died, 19 (3.6\%) had recurrence of GC, and $40(7.6 \%)$ died of other causes. Thirty-two patients (6.0\%) died of benign diseases, such as cardiac, pulmonary, hepatic, and renal disease.

Patients' characteristics and surgical outcomes are shown in Table 1. In this study, PCs were defined as grade 2 or higher events according to the Clavien-Dindo classification that occurred within 30 days after gastrectomy [9]. In addition, PCs were classified into either abdominal or non-abdominal complications.

Next, we identified patients who had comorbidities that were risk factor for PCs. They were divided into two groups: patients underwent insufficient LND and those underwent sufficient LND. Insufficient LND included both insufficient $\mathrm{D} 1+$ dissection in pathological stage IA cancer with submucosal invasion and insufficient D2 dissection in pathological stage IB or more advanced cancer. We compared clinical outcomes between the patients who underwent insufficient LND and those underwent sufficient LND.

\section{Statistical analysis}

All variables were classified into two categories and were compared with the use of the chi-square test or Fisher's exact test, as appropriate. Multivariate analysis was carried out by binary logistic multiple regression testing using dummy variables. Seven patients (1.3\%) were excluded from the multivariate analysis because of missing data. Survival was measured from the date of performing LAG to the latest follow-up date or the date of death. Kaplan-Meier curves were plotted to assess the effect of insufficient LND for patients with any risky comorbidity on survival. Different curves of survival were compared using the log-rank test. P values of $<0.05$ were considered to indicate statistical significance. All analyses were performed with the statistical software package SPSS 20 (SPSS Japan Inc., Tokyo, Japan). 
Table 1 Patients' characteristics and surgical outcomes

\begin{tabular}{|c|c|}
\hline & $\mathrm{n} \%$ \\
\hline \multicolumn{2}{|l|}{ Gender } \\
\hline Male & $380(71.8)$ \\
\hline Female & $149(28.2)$ \\
\hline Age mean $\pm S D$ & $64.9 \pm 11.5$ \\
\hline Body mass index $\left(\mathrm{kg} / \mathrm{m}^{2}\right)$ mean $\pm \mathrm{SD}$ & $22.9 \pm 3.1$ \\
\hline Comorbidities & $326(61.6)$ \\
\hline Heart disease & $50(9.5)$ \\
\hline Ischemic disease & $24(4.5)$ \\
\hline Arrhythmia & $24(4.5)$ \\
\hline Congenital cardiac failure & $3(0.6)$ \\
\hline Others & $7(1.3)$ \\
\hline CNS disease & $39(7.4)$ \\
\hline Cerebrovascular disease & $30(5.7)$ \\
\hline Neurodegenerative disease & $6(1.1)$ \\
\hline Others & $3(0.6)$ \\
\hline Liver disease & $21(4.0)$ \\
\hline Liver cirrhosis & $8(1.5)$ \\
\hline Chronic hepatitis & $13(2.4)$ \\
\hline Renal dysfunction ${ }^{a)}$ & $54(10.2)$ \\
\hline Pulmonary dysfunction & $124(23.4)$ \\
\hline Restrictive pulmonary dysfunction ${ }^{\text {b) }}$ & $25(4.7)$ \\
\hline COPD & $112(21.2)$ \\
\hline Diabetes mellitus & $67(12.7)$ \\
\hline Hypertension & $184(34.8)$ \\
\hline Other disease & $45(8.5)$ \\
\hline Anemia $^{c}$ & $131(24.8)$ \\
\hline Hyoalbuminemia $^{\text {d) }}$ & $8(1.5)$ \\
\hline \multicolumn{2}{|l|}{ Type of gastrectomy } \\
\hline Total & $78(14.7)$ \\
\hline Proximal & $34(6.4)$ \\
\hline Distal & $417(78.8)$ \\
\hline \multicolumn{2}{|l|}{ Extent of LND } \\
\hline D1 & $4(0.8)$ \\
\hline $\mathrm{D} 1+$ & $448(84.7)$ \\
\hline D2 & $77(14.6)$ \\
\hline Combined resection & $54(10.2)$ \\
\hline Gallbladder & $39(7.4)$ \\
\hline Spleen & $13(2.5)$ \\
\hline Intestine or colon & $2(0.4)$ \\
\hline Operating time $(\mathrm{min})$ mean $\pm \mathrm{SD}$ & $287 \pm 75$ \\
\hline Bleeding (g) median (range) & $72(0-2492)$ \\
\hline
\end{tabular}

Table 1 Patients' characteristics and surgical outcomes (Continued)

\begin{tabular}{l}
\hline Pathological tumor stage \\
II \\
III (82.8) \\
\hline SD standard deviation, CNS central nervous system. \\
COPD: chronic obstructive pulmonary disease, LND: lymph node dissection \\
a) serum creatinine concentration higher than the upper limit of normal at our \\
hospital, $>1.10$ in males and $>0.80$ in females. \\
b) predicted vital capacity $<80 \%$. \\
c) decreased hemoglobin, $<13 \mathrm{~g} / \mathrm{dL}$ in males and $<12 \mathrm{~g} / \mathrm{dL}$ in females. \\
d) decreased serum albumin $<3.5 \mathrm{~g} / \mathrm{dL}$.
\end{tabular}

\section{Results}

A total of $87(16.4 \%)$ patients had PCs. There was no PC-related death. Overall, 66 (12.5\%) patients had abdominal complications, 5 (0.9\%) had cardiac complications, 10 (1.9\%) had pulmonary complications, and 15 (2.8\%) had other complications. The details of the PCs are shown in Table 2. As for surgical factors, D2 LND, D1+ LND, and D1 LND were performed in 77 (14.6\%), 448 (84.7\%), and 4 $(0.8 \%)$ patients, respectively. Total gastrectomy, proximal gastrectomy, and distal gastrectomy were performed in 78 (14.7\%), 34 (6.4\%), and 417 (78.8\%) patients.

\section{All PCs}

On univariate analysis, PCs were significantly associated with many factors: male gender, higher age ( $\geq 75$ years), heart disease, CNS disease, liver disease, renal dysfunction, restrictive pulmonary dysfunction, anemia, regular use of steroids, total gastrectomy, combined resection of other organ (except gallbladder), extended operating time ( $\geq 300$ minutes), and higher operative bleeding volume $(\geq 300 \mathrm{~g})$ (Table 3$)$. Only 5 patients $(0.9 \%)$ received transfusion, and transfusion was not assessed in this study. Next, we evaluated independent risk factors for PCs using a multivariate model adjusted for all of the above risk factors (Table 4). Finally, both liver disease and heart disease were independent risk factors significantly related to $\mathrm{PCs}$ on multivariate analysis (odds ratio $[\mathrm{OR}]=3.25$, 95\% confidential interval $[\mathrm{CI}]: 1.18-8.91, \mathrm{p}=0.022$; $\mathrm{OR}=2.36,95 \% \mathrm{CI}: 1.17-4.76, \mathrm{p}=0.017$, respectively). The following factors showed a trend toward being risk factors on multivariate analysis: CNS disease $(\mathrm{OR}=2.24$, 95\% CI: $1.00-5.01, \mathrm{p}=0.050)$, renal dysfunction $(\mathrm{OR}=2.01$, 95\% CI: 0.98-4.13, $\mathrm{p}=0.058)$, male gender $(\mathrm{OR}=1.75,95 \%$ CI: $0.93-3.29, \mathrm{p}=0.082)$, higher age $(\mathrm{OR}=1.70,95 \% \mathrm{CI}$ : $0.95-3.03, \mathrm{p}=0.075)$, combined resection $(\mathrm{OR}=2.85,95 \%$ CI: 0.88-9.27, $\mathrm{p}=0.081$ ), and extended operating time $(\mathrm{OR}=1.61,95 \% \mathrm{CI}: 0.95-2.73, \mathrm{p}=0.079)$.

\section{Subcategorized PCs}

For analysis, PCs were subcategorized into abdominal and non-abdominal (cardiac, pulmonary, etc.) PCs. Abdominal 
Table 2 Postoperative complications

\begin{tabular}{|c|c|c|}
\hline & $\mathrm{n} \%$ & Grade $2 / 3 / 4 / 5$ \\
\hline Total & $87(16.4)$ & $48 / 34 / 5 / 0$ \\
\hline Abdominal & $66(12.5)$ & $31 / 33 / 2 / 0$ \\
\hline Anastomotic leakage & $8(1.5)$ & $0 / 7 / 1 / 0$ \\
\hline Pancreatic fistula & $5(0.9)$ & $1 / 4 / 0 / 0$ \\
\hline Abdominal abscess & $14(2.6)$ & $4 / 10 / 0 / 0$ \\
\hline Anastomotic stenosis & $14(2.6)$ & $3 / 11 / 0 / 0$ \\
\hline lleus & $9(1.7)$ & $5 / 3 / 1 / 0$ \\
\hline Gastric stasis & $5(0.9)$ & $5 / 0 / 0 / 0$ \\
\hline Postoperative bleeding & $4(0.8)$ & $3 / 1 / 0 / 0$ \\
\hline Ascites & $4(0.8)$ & $3 / 1 / 0 / 0$ \\
\hline Cholecystitis & $2(0.4)$ & $1 / 1 / 0 / 0$ \\
\hline Cholerrhagia & $1(0.2)$ & $0 / 1 / 0 / 0$ \\
\hline Reflux esophagitis & $2(0.4)$ & $2 / 0 / 0 / 0$ \\
\hline Enteritis & $1(0.2)$ & $1 / 0 / 0 / 0$ \\
\hline Wound infection & $6(1.1)$ & $6 / 0 / 0 / 0$ \\
\hline Non-abdominal & $30(0.9)$ & $25 / 2 / 3 / 0$ \\
\hline Ischemic attack & $1(0.2)$ & 0/0/1/0 \\
\hline Arrhythmia & $4(0.8)$ & $4 / 0 / 0 / 0$ \\
\hline Pneumonia & $6(1.1)$ & $5 / 1 / 0 / 0$ \\
\hline ARDS & $2(0.4)$ & $0 / 0 / 2 / 0$ \\
\hline Atelectasis & $2(0.4)$ & $2 / 0 / 0 / 0$ \\
\hline Urinary tract infection & $2(0.4)$ & $2 / 0 / 0 / 0$ \\
\hline Infection of venous catheter & $1(0.2)$ & $1 / 0 / 0 / 0$ \\
\hline Deep vein thrombosis & $1(0.2)$ & $0 / 1 / 0 / 0$ \\
\hline Cerebral bleeding & $1(0.2)$ & 0/0/1/0 \\
\hline Delirium & $12(2.3)$ & $12 / 0 / 0 / 0$ \\
\hline
\end{tabular}

ARDS acute respiratory distress syndrome.

PCs occurred in $75.9 \%$ of the patients with PCs. Abdominal PCs were significantly associated with many factors on univariate analysis (Table 3). Multivariate analysis showed 3 independent predictors of abdominal PCs: liver disease $(\mathrm{OR}=3.10,95 \% \mathrm{CI}: 1.13-8.47, \mathrm{p}=0.028)$, heart disease $(\mathrm{OR}=2.40,95 \% \mathrm{CI}: 1.20-4.82, \mathrm{p}=0.013)$, and renal dysfunction $(\mathrm{OR}=2.13,95 \% \mathrm{CI}: 1.06-4.29, \mathrm{p}=0.035)$. Extended operating time and higher operative bleeding were not significant predictors on multivariate analysis $(\mathrm{OR}=1.57,95 \% \mathrm{CI}: 0.93-2.64, \mathrm{p}=0.093 ; \mathrm{OR}=1.22,95 \%$ CI: $0.54-2.76, \mathrm{p}=0.64$, respectively) (Table 4 ).

Non-abdominal PCs were also significantly associated with many factors on univariate analysis (Table 3). Heart disease was also an independent risk factor for nonabdominal PCs $(\mathrm{OR}=2.31,95 \% \mathrm{CI}: 1.15-4.64, \mathrm{p}=0.019)$ on multivariate analysis. Three other factors were independent predictors of non-abdominal PCs: higher age $(\mathrm{OR}=1.84,95 \% \mathrm{CI}: 1.04-3.26, \mathrm{p}=0.036)$, regular use of steroids $(\mathrm{OR}=4.47,95 \% \mathrm{CI}: 1.04-19.3, \mathrm{p}=0.045)$, and extended operating time $(\mathrm{OR}=1.71,95 \% \mathrm{CI}: 1.02-2.86$, $\mathrm{p}=0.043)($ Table 4).

\section{Relation between PCs and severity of comorbidities}

The severity of each comorbidity was not significantly related to an increased incidence of PCs, although a high rate of PCs was found in patients with liver cirrhosis (Table 5).

\section{Impact of insufficient LND on PCs and survival of patients with any risky comorbidity}

We assessed the impact of insufficient LND (as defined in the Methods section) on PCs and survival in patients with the following risky comorbidities: heart disease, CNS disease, liver disease, renal dysfunction, and restrictive pulmonary dysfunction, all of which were significantly associated with PCs. A total of 149 patients (28\% of all patients) had these risky comorbidities, and 42 (28\%) of these patients underwent insufficient LND. The characteristics of the patients included in this portion of the study are shown in Table 6. The patients who underwent insufficient LND had a more advanced stage of GC $(\mathrm{p}<0.001)$. The incidences of all PCs and of abdominal PCs were similar in the patients who underwent insufficient LND and those who underwent sufficient LND (29\% vs 30\%, $\mathrm{p}=0.87 ; 19 \%$ vs $25 \%, \mathrm{p}=0.42$, respectively). However, the incidence of non-abdominal PCs was significantly higher in the patients who underwent insufficient LND than in those who underwent sufficient LND (21\% vs $8 \%, p=0.028$ ) (Table 6). The overall survival rate was slightly, but not significantly lower in patients who received insufficient LND (60.6\% vs $79.0 \%, \mathrm{p}=0.24)$. However, GC-specific survival was similar in the two groups $(90.6 \%$ vs $94.4 \%, \mathrm{p}=0.25)$, regardless of the fact that patients who underwent insufficient LND had a significantly more advanced stage of GC than those who underwent sufficient LND (Table 7).

\section{Discussion}

Our results showed that heart, CNS, liver, renal, and pulmonary comorbidities or dysfunctions were risk factors for PCs after radical gastrectomy. Heart disease and liver disease were independent risk factors for PCs in the present study, consistent with the results of a previous study of gastrectomy with D2 LND by Jeong et al. [2]. Heart disease and liver disease might be common risk factors after gastrectomy. However, Jeong et al. did not mention renal or pulmonary dysfunction, and the rates of comorbidities such as heart disease (4.6\%) and neurological disease $(2.2 \%)$ were lower than those in our study. Moreover, the rate of laparoscopic surgery was only $9.0 \%$ in their study. Another study found that liver cirrhosis and hypertension were independent risk factors for PCs in patients $\geq 70$ years of age who underwent gastrectomy [5]. 
Table 3 Univariate analysis for risk factors of PCs in LAG

\begin{tabular}{|c|c|c|c|c|c|c|c|c|}
\hline & & & All & & Abdc & & Non-ab & minal \\
\hline & & $n$ & n (\%) & $p$ & n (\%) & $p$ & n (\%) & $p$ \\
\hline Gender & male & 380 & $71(18.7)$ & 0.027 & $53(13.9)$ & 0.10 & $27(7.1)$ & 0.023 \\
\hline & female & 149 & $16(10.7)$ & & $13(10.8)$ & & $3(2.0)$ & \\
\hline Age & $\geq 75$ & 117 & $34(29.0)$ & $<0.001$ & $27(23.1)$ & $<0.001$ & $12(11.4)$ & 0.015 \\
\hline & $<75$ & 412 & $53(12.9)$ & & $39(9.5)$ & & $18(4.4)$ & \\
\hline Body mass index & $\geq 25\left(\mathrm{~kg} / \mathrm{m}^{2}\right)$ & 136 & $18(13.2)$ & 0.24 & $15(11.0)$ & 0.55 & $6(4.4)$ & 0.46 \\
\hline & $<25$ & 393 & 69 (17.6) & & $51(13.0)$ & & $24(6.1)$ & \\
\hline Heart disease & yes & 50 & $18(36.0)$ & $<0.001$ & $13(26.0)$ & 0.002 & $10(20.0)$ & $<0.001$ \\
\hline & no & 479 & $69(14.4)$ & & $53(11.1)$ & & $20(4.1)$ & \\
\hline CNS disease & yes & 39 & $13(33.3)$ & 0.003 & $10(25.6)$ & 0.020 & $7(17.9)$ & 0.004 \\
\hline & no & 490 & $74(15.1)$ & & $56(11.4)$ & & $23(4.7)$ & \\
\hline Liver disease & yes & 21 & $8(38.1)$ & 0.013 & $8(38.1$ & $<0.001$ & $2(9.5)$ & 0.34 \\
\hline & no & 508 & 79 (15.6) & & $58(11.4)$ & & $28(5.5)$ & \\
\hline Diabetes mellitus & yes & 67 & $12(17.9)$ & 0.73 & $10(14.9)$ & 0.52 & $5(7.5)$ & 0.57 \\
\hline & no & 462 & 75 (16.2) & & $56(12.1)$ & & $25(5.4)$ & \\
\hline Hypertension & yes & 184 & $34(18.5)$ & 0.36 & $25(13.6$ & 0.57 & $13(7.1)$ & 0.31 \\
\hline & no & 345 & $53(15.4)$ & & 41 (11.9) & & $17(4.9)$ & \\
\hline Renal dysfunction & yes & 54 & $17(31.5)$ & 0.002 & $14(25.9)$ & 0.002 & $6(11.1)$ & 0.11 \\
\hline & no & 475 & $70(14.7)$ & & $52(12.3)$ & & $24(5.1)$ & \\
\hline Restrictive pulmonary dysfunction & yes & 25 & $10(40.0)$ & 0.003 & $7(28.0)$ & 0.026 & $6(24.0)$ & 0.001 \\
\hline & no & 498 & $75(15.0)$ & & $58(13.2)$ & & $23(4.6)$ & \\
\hline & not evaluated & 6 & 2 & & 1 & & 1 & \\
\hline COPD & yes & 112 & $17(15.2)$ & 0.73 & $11(9.8)$ & 0.35 & $8(7.1)$ & 0.41 \\
\hline & no & 411 & $68(16.5)$ & & $54(13.1)$ & & $21(5.1)$ & \\
\hline & not evaluated & 6 & 2 & & 1 & & 1 & \\
\hline Anemia & yes & 131 & $29(22.1)$ & 0.043 & $22(16.8)$ & 0.085 & $12(9.2)$ & 0.047 \\
\hline & no & 398 & $58(14.6)$ & & $44(11.1)$ & & $18(4.5)$ & \\
\hline Hypoalbuminemia & yes & 8 & $3(37.5)$ & 0.13 & $2(25.0)$ & 0.26 & $2(25.0)$ & 0.071 \\
\hline & no & 519 & $84(16.2)$ & & $64(12.3)$ & & $28(5.4)$ & \\
\hline & not evaluated & 2 & 0 & & 0 & & 0 & \\
\hline Regular use of steroid & yes & 9 & $4(44.4)$ & 0.045 & $2(22.2)$ & 0.31 & $2(22.2)$ & 0.087 \\
\hline & no & 520 & $83(16.0)$ & & $64(12.3)$ & & $28(5.4)$ & \\
\hline Type of resection & total or proximal & 112 & $29(25.9)$ & 0.002 & $20(17.9)$ & 0.052 & $13(11.6)$ & 0.002 \\
\hline & distal & 417 & $58(13.9)$ & & $46(12.4)$ & & $17(4.1)$ & \\
\hline Extent of lymph node dissection & D2 & 77 & $15(19.5)$ & 0.44 & $10(13.0)$ & 0.88 & $5(6.5)$ & 0.79 \\
\hline & $\mathrm{D} 1+$ or $\mathrm{D} 1$ & 452 & $72(15.9)$ & & $56(12.4)$ & & $25(5.5)$ & \\
\hline Combined resection & yes & 16 & $6(37.5)$ & 0.033 & $4(25.0)$ & 0.13 & $2(12.5)$ & 0.23 \\
\hline & no or gallbladder & 513 & $81(15.8)$ & & $62(12.1)$ & & $28(5.5)$ & \\
\hline Operating time & $\geq 300$ (min) & 253 & $52(20.6)$ & 0.015 & 39 (15.4) & 0.050 & $19(7.5)$ & 0.080 \\
\hline & $<300$ & 276 & $35(12.7)$ & & $27(9.8)$ & & $11(4.0)$ & \\
\hline Estimated bleeding & $\geq 500(\mathrm{~g})$ & 43 & $12(27.9)$ & 0.031 & $10(23.3)$ & 0.026 & $4(9.3)$ & 0.28 \\
\hline & $<500$ & 485 & $74(15.2)$ & & $56(11.5)$ & & $25(5.2)$ & \\
\hline & unknown & 1 & 1 & & 0 & & 1 & \\
\hline
\end{tabular}


Table 4 Multivariate analysis of risk factors for PCs in LAG

\begin{tabular}{|c|c|c|c|c|c|c|c|c|c|}
\hline & & All PCs & & & odominal $\mathrm{F}$ & & & -abdomina & \\
\hline & OR & $95 \% \mathrm{Cl}$ & $p$ & OR & $95 \% \mathrm{Cl}$ & $p$ & OR & $95 \% \mathrm{Cl}$ & $p$ \\
\hline Male gender & 1.75 & $0.93-3.29$ & 0.082 & 1.70 & $0.91-3.17$ & 0.099 & 1.57 & $0.86-2.89$ & 0.15 \\
\hline Higher age $(\geq 75)$ & 1.70 & $0.95-3.03$ & 0.075 & 1.66 & $0.93-2.96$ & 0.086 & 1.84 & $1.04-3.26$ & 0.036 \\
\hline Heart disease & 2.36 & $1.17-4.76$ & 0.017 & 2.40 & $1.20-4.82$ & 0.013 & 2.31 & $1.15-4.64$ & 0.019 \\
\hline CNS disease & 2.24 & $1.00-5.01$ & 0.050 & 2.11 & $0.94-4.73$ & 0.070 & 1.99 & $0.88-4.49$ & 0.097 \\
\hline Liver disease & 3.25 & $1.18-8.91$ & 0.022 & 3.10 & $1.13-8.47$ & 0.028 & & & \\
\hline Renal dysfunction & 2.01 & $0.98-4.13$ & 0.058 & 2.13 & $1.06-4.29$ & 0.035 & & & \\
\hline Restrictive pulmonary dysfunction & 2.08 & $0.81-5.34$ & 0.13 & 1.95 & $0.76-4.99$ & 0.16 & 2.12 & $0.83-5.42$ & 0.12 \\
\hline Anemia & 0.93 & $0.51-1.69$ & 0.81 & 1.04 & $0.58-1.85$ & 0.90 & 1.02 & $0.57-1.83$ & 0.95 \\
\hline Hypoalbuminemia & & & & & & & 1.53 & $0.21-11.2$ & 0.67 \\
\hline Regular use of steroids & 2.93 & $0.58-14.8$ & 0.19 & & & & 4.47 & $1.04-19.3$ & 0.045 \\
\hline Total or proximal gastrectomy & 1.39 & $0.76-2.55$ & 0.29 & 1.52 & $0.85-2.73$ & 0.16 & 1.73 & $0.99-3.00$ & 0.052 \\
\hline Combined resection & 2.85 & $0.88-9.27$ & 0.081 & & & & & & \\
\hline Extended operating time ( $\geq 300 \mathrm{~min})$ & 1.61 & $0.95-2.73$ & 0.079 & 1.57 & $0.93-2.64$ & 0.093 & 1.71 & $1.02-2.86$ & 0.043 \\
\hline Higher operative bleeding ( $\geq 500 \mathrm{~g}$ ) & 1.10 & $0.48-2.52$ & 0.83 & 1.22 & $0.54-2.76$ & 0.64 & & & \\
\hline
\end{tabular}

PCs postoperative complications, LAG laparoscopy-assisted gastrectomy.

Most patients underwent D1+ LND and distal gastrectomy in our study, because the Japanese guidelines recommend LAG for the treatment of early GC. Therefore, our results would most likely differ somewhat from those of similar studies performed in Western countries owing to differences in the most common sites of GC and the disease stage at diagnosis as compared with Japan.

In previous studies of only LAG in patients with mainly early gastric cancer, higher age ( $\geq 60$ years), male gender of the patient, and type of resection or reconstruction procedure were predictors of local PCs, and inadequate

Table 5 Relationship between severity of each comorbidity and PCs

\begin{tabular}{llrcl}
\hline Comorbidity & Classification by severity & \multicolumn{3}{c}{ PCs } \\
\cline { 3 - 5 } & & $\mathbf{n}$ & $\mathbf{n}(\%)$ & $\mathbf{p}$ \\
\hline Heart disease & surgical or interventional & 19 & $5(26)$ & 0.26 \\
& only medication & 31 & $13(42)$ & \\
COPD & stage 3 or 4* & 8 & $0(0)$ & 0.60 \\
& stage 1 or 2 & 104 & $17(16)$ & \\
CNS disease & paralysis & 8 & $2(25)$ & 0.69 \\
& no paralysis & 31 & $11(35)$ & \\
Liver disease & cirrhosis & 8 & $5(63)$ & 0.16 \\
& hepatitis & 13 & $3(23)$ & \\
Renal dysfunction & dialysis & 5 & $2(40)$ & 0.65 \\
& no dialysis & 49 & $15(31)$ & \\
Diabetes mellitus & regular use of insulin & 16 & $4(25)$ & 0.46 \\
& oral medication & 51 & $8(16)$ & \\
\hline
\end{tabular}

*The stage of COPD is defined by Global Initiative for Chronic Obstructive Lung Disease. experience of the operator was a predictor of systemic PCs [3,4]. Higher age was not a significant predictor of PCs in other studies of LAG [10-13], while higher age was significantly associated with non-abdominal PCs in this study. In the present study, 4 surgeons qualified in LAG performed all LAG procedures. The experience of the surgeons thus did not affect clinical outcomes. Our study had several limitations. Most important, it was a single-center study performed by experts in LAG. Our results thus might not be applicable to general hospitals. A pooled analysis or a multicenter study involving surgeons with various degrees of experience is necessary to identify common risk factors for gastrectomy.

In three studies of D2 LND including many patients who underwent OG, multiple-organ resection, advanced disease stage, extended operating time ( $\geq 180$ or $200 \mathrm{mi}-$ nutes), higher age ( $\geq 50$ years), male gender, higher BMI $(\geq 25)$, and type of reconstruction were significant independent predictors of PCs $[2,14,15]$. In a randomized clinical trial of OG with D2 or more extended LND, higher age ( $>65$ years), pancreatectomy, and extended operating time (>297 minutes) were independent risk factors for PCs [16]. In another study of open gastrectomy with various extents of lymph-node dissection, splenectomy or an extended operative time ( $\geq 360$ minutes) was a risk factor for abdominal PCs [17].

Obesity is an established operative risk factor, but patients with a BMI of $\geq 30$, defined as obese by the WHO, are uncommon in Asia. Obesity has therefore been an uncertain predictor of PCs in patients who undergo LAG [18-21]. Diabetes mellitus is a known risk factor for PCs after pancreaticoduodenectomy and 
Table 6 Comparison between insufficient LND and sufficient LND in patients with any risky comorbidity

\begin{tabular}{|c|c|c|c|c|}
\hline & & $\begin{array}{c}\text { Insufficient LND } \\
\qquad \begin{array}{c}n=42 \\
n(\%)\end{array}\end{array}$ & $\begin{array}{c}\text { Sufficient LND } \\
\begin{array}{c}n=107 \\
n(\%)\end{array}\end{array}$ & $\mathrm{p}$ \\
\hline \multirow[t]{2}{*}{ Age } & $\geq 75$ & $18(43)$ & $45(42)$ & 0.93 \\
\hline & $<75$ & $24(57)$ & $62(58)$ & \\
\hline \multirow[t]{2}{*}{ Gender } & male & $34(81)$ & $76(71)$ & 0.22 \\
\hline & female & $8(19)$ & $34(29)$ & \\
\hline \multirow[t]{3}{*}{ Tumor stage } & । & $22(52)$ & $96(90)$ & $<0.001$ \\
\hline & $\|$ & $14(33)$ & $7(7)$ & \\
\hline & III & $6(14)$ & $4(4)$ & \\
\hline \multirow[t]{3}{*}{ LND } & D1 & $2(5)$ & $1(0.9)$ & 0.015 \\
\hline & $\mathrm{D} 1+$ & $40(95)$ & $91(85)$ & \\
\hline & D2 & $0(0)$ & $15(14)$ & \\
\hline \multirow{3}{*}{$\begin{array}{l}\text { No. of risky } \\
\text { comorbidity }\end{array}$} & 1 & $32(76)$ & $83(78)$ & 0.73 \\
\hline & 2 & $9(21)$ & $19(18)$ & \\
\hline & $\geq 3$ & $1(2)$ & $5(5)$ & \\
\hline \multicolumn{2}{|l|}{ All PCs } & $12(29)$ & $32(30)$ & 0.87 \\
\hline \multicolumn{2}{|c|}{ Abdominal PCs } & $8(19)$ & $27(25)$ & 0.42 \\
\hline \multicolumn{2}{|c|}{ Non-abdominal PCs } & $9(21)$ & $9(8)$ & 0.028 \\
\hline
\end{tabular}

hepatectomy [22,23], while Jeong et al. found no relation between diabetes mellitus and PCs after gastrectomy [5]. Preoperative strict diabetic control by diabetologists for about 2 weeks in patients with severe diabetes mellitus in our hospital might have resulted in the favorable postoperative course. COPD is a risk factor for postoperative pulmonary complications after non-thoracic surgery [24]. COPD was not associated with postoperative pulmonary complications in our study or in a previous study including patients who received open gastrectomy [25].

Table 7 OS and DSS in patients with any risky comorbidity

\begin{tabular}{|c|c|c|c|c|c|}
\hline & & $\begin{array}{l}\text { 5-year } \\
\text { OS (\%) }\end{array}$ & $p$ & $\begin{array}{c}\text { 5-year } \\
\text { DSS (\%) }\end{array}$ & $p$ \\
\hline \multirow[t]{2}{*}{ Age } & $<75$ & 77.3 & 0.069 & 92.6 & 0.94 \\
\hline & $\geq 75$ & 44.9 & & 94.4 & \\
\hline \multirow[t]{2}{*}{ Gender } & male & 76.5 & 0.43 & 100.0 & 0.22 \\
\hline & female & 68.5 & & 80.8 & \\
\hline \multirow[t]{3}{*}{ Tumor stage } & । & 81.8 & $<0.001^{\mathrm{a}}$ & 100.0 & $<0.001^{\mathrm{a}, \mathrm{b}}$ \\
\hline & $\|$ & 64.6 & $0.006^{c}$ & 80.8 & $0.022^{c}$ \\
\hline & III & 25.0 & & 42.2 & \\
\hline \multirow{2}{*}{$\begin{array}{l}\text { No. of risky } \\
\text { comorbidity }\end{array}$} & 1 & 73.3 & 0.19 & 92.8 & 0.61 \\
\hline & $\geq 2$ & 45.1 & & 95.0 & \\
\hline \multirow[t]{2}{*}{ LND } & sufficient & 79.0 & 0.24 & 94.4 & 0.25 \\
\hline & insufficient & 60.6 & & 90.6 & \\
\hline
\end{tabular}

OS; overall survival, DSS; disease-specific survival.

a stage I vs III, ${ }^{\text {b }}$ stage I vs II, ' stage II vs III.
Preoperative smoking cessation for about 3 to 4 weeks in all patients and breathing exercises in patients with severe COPD might have contributed to the low incidence of pulmonary complications (10 patients, 1.8\%), and $8(1.5 \%)$ patients with 3 or more severe COPDs had no pulmonary complications in this study.

Nomograms established from preoperative data can facilitate the design of treatment strategies, but require a large volume of data from multiple centers. The Charlson comorbidity index (CCI) was developed to predict 10-year mortality for patients with a range of comorbidities [26]. Park et al. showed that the age-adjusted CCI was a useful predictor of systemic complications after LAG [27]. EPASS and POSSUM predict the risks of mortality and morbidity after various operations, and the latter has been employed in patients undergoing gastrectomy [6,7]. However, these systems have not been routinely used in clinical practice, and many surgeons base treatment strategies on the severity of comorbidities or age of the patient. Clinically, reduced insufficient LND is often performed in patients with severe comorbidity or higher age, although criteria defining the need for more conservative procedures remain unclear. The indications for insufficient LND in risky patients were decided by consensus among a team of gastrointestinal surgeons in our hospital and were primarily based on the general condition of risky patients; we had no predefined criteria for such indications. We performed at least D1+ LND in risky patients who had a preoperative diagnosis of advanced GC. Insufficient LND did not reduce PCs in patients with risky comorbidities. In contrast, cardiac or pulmonary PCs increased in this study. However, if all patients had undergone sufficient LND, more PCs might have occurred. In addition, insufficient LND did not significantly shorten GC-specific survival in patients with any risky comorbidity. Insufficient LND, such as D1+ LND for advanced cancer, may thus be permissible in high-risk patients. A prospective randomized controlled trial would be the most reliable means of objectively evaluating the advantages and disadvantages of insufficient LND, but would be risky to perform in patients with severe comorbidities. A multicenter study or a pooled analysis is considered a better means of resolving this issue in the future. In the present study, the severity of comorbidities was not significantly related to the incidence of PCs. This finding might be attributed to the fact that few patients with severe comorbidities were allowed to receive prolonged general anesthesia. In such patients, we performed local resection with limited sampling of lymph nodes, endoscopic resection without LND, or sometimes withheld anticancer treatments.

\section{Conclusions}

Heart, CNS, liver, renal, and pulmonary comorbidities or dysfunctions were risk factors for PCs after LAG in 
patients with GC. Heart disease and liver disease were independent risk factors for PCs. In high-risk patients, insufficient LND did not decrease PCs, but had no negative impact on GC-specific survival. Insufficient LND, such as D1+ LND for advanced GC, might thus be permissible in this subgroup of patients.

\section{Consent}

Written informed consent was obtained from the patients or their guardian/parent/next of kin for this postoperative research.

\section{Competing interests}

The authors declare that they have no competing interests.

\section{Authors' contributions}

Ml was responsible for drafting the manuscript. KK, KK, SO and HS contributed to data analysis and interpretation. All authors read and approved the final manuscript.

\section{Acknowledgements}

We thank K. Watanabe for inputting information of patients.

\section{Author details}

'Department of Surgical Oncology, Tokyo Medical and Dental University, Tokyo, Japan. ${ }^{2}$ Department of Minimally Invasive Surgery, Tokyo Medical and Dental University, Tokyo, Japan.

Received: 14 February 2014 Accepted: 11 November 2014

Published: 22 November 2014

\section{References}

1. Ferlay J, Shin HR, Bray F, Forman D, Mathers C, Parkin DM: Estimates of worldwide burden of cancer in: GLOBOCAN 2008. Int J Cancer 2010, 127:2893-2917.

2. Jeong SH, Ahn HS, Yoo MW, Cho JJ, Lee HJ, Kim HH, Lee KU, Yang HK: Increased morbidity rates in patients with heart disease or chronic liver disease following radical gastric surgery. I Surg Oncol 2010, 101:200-204.

3. Kim MC, Kim W, Kim HH, Ryu SW, Ryu SY, Song KY, Lee HJ, Cho GS, Han SU, Hyung WJ, Korean Laparoscopic Gastrointestinal Surgery Study (KLASS) Group: Risk factors associated with complication following laparoscopy-assisted gastrectomy for gastric cancer: a large-scale korean multicenter study. Ann Surg Oncol 2008, 15:2692-2700.

4. Kim W, Song KY, Lee HJ, Han SU, Hyung WJ, Cho GS: The impact of comorbidity on surgical outcomes in laparoscopy-assisted distal gastrectomy: a retrospective analysis of multicenter results. Ann Surg 2008, 248:793-799.

5. Hwang SH, Park do J, Jee YS, Kim HH, Lee HJ, Yang HK, Lee KU: Risk factors for operative complications in elderly patients during laparoscopy-assisted gastrectomy. J Am Coll Surg. 2009, 208:186-192.

6. Haga Y, Ikejiri K, Wada Y, Takahashi T, Ikenaga M, Akiyama N, Koike S, Koseki M, Saitoh T: A multicenter prospective study of surgical audit systems. Ann Surg 2011, 253:194-201

7. Dutta S, Horgan PG, McMillan DC: POSSUM and its related models as predictors of postoperative mortality and morbidity in patients undergoing surgery for gastro-oesophageal cancer: a systematic review. World J Surg 2010, 34:2076-2082.

8. Japanese Gastric Cancer Association: Gastric cancer treatment guidelines for doctor's reference, version 2. Tokyo (in Japanese): Kanehara Press; 2004.

9. Dindo D, Demartines N, Clavien PA: Classification of surgical complications: a new proposal with evaluation in a cohort of 6336 patients and results of a survey. Ann Surg 2004, 240:205-213.

10. Kim HH, Hyung WJ, Cho GS, Kim MC, Han SU, Kim W, Ryu SW, Lee HJ, Song $\mathrm{KY}$ : Morbidity and mortality of laparoscopic gastrectomy versus open gastrectomy for gastric cancer: an interim report-a phase III multicenter, prospective, randomized Trial (KLASS Trial). Ann Surg 2010, 251:417-420.

11. Tokunaga M, Hiki N, Fukunaga T, Miki A, Ohyama S, Seto Y, Yamaguchi T: Does age matter in the indication for laparoscopy-assisted gastrectomy? J Gastrointest Surg 2008, 12:1502-1507.
12. Kunisaki C, Makino H, Takagawa R, Oshima T, Nagano Y, Ono HA, Akiyama H, Shimada H: Efficacy of laparoscopy-assisted distal gastrectomy for gastric cancer in the elderly. Surg Endosc 2009, 23:377-383.

13. Yamada H, Kojima K, Inokuchi M, Kawano T, Sugihara K: Laparoscopy-assisted gastrectomy in patients older than 80. J Surg Res 2010, 161:259-263.

14. Park DJ, Lee HJ, Kim HH, Yang HK, Lee KU, Choe KJ: Predictors of operative morbidity and mortality in gastric cancer surgery. Br J Surg 2005, 92:1099-1102.

15. Jeong O, Park YK, Ryu SY, Kim YJ: Effect of age on surgical outcomes of extended gastrectomy with $\mathrm{D} 2$ lymph node dissection in gastric carcinoma: prospective cohort study. Ann Surg Oncol 2010, 17:1589-1596. B.

16. Kodera Y, Sasako M, Yamamoto S, Sano T, Nashimoto A, Kurita A, Gastric Cancer Surgery Study Group of Japan Clinical Oncology Group: Identification of risk factors for the development of complications following extended and superextended lymphadenectomies for gastric cancer. Br J Surg 2005, 92:1103-1109.

17. Ichikawa D, Kurioka H, Yamaguchi T, Koike H, Okamoto K, Otsuji E, Shirono K, Shioaki Y, Ikeda E, Mutoh F, Yamagishi H: Postoperative complications following gastrectomy for gastric cancer during the last decade. Hepatogastroenterology 2004, 51:613-617.

18. Yoshikawa K, Shimada M, Kurita N, Iwata T, Nishioka M, Morimoto S, Miyatani T, Komatsu M, Mikami C, Kashihara H: Visceral fat area is superior to body mass index as a predictive factor for risk with laparoscopyassisted gastrectomy for gastric cancer. Surg Endosc 2011, 25:3825-3830.

19. Kawamura H, Tanioka T, Funakoshi T, Takahashi M: Surgical effects of obesity on laparoscopy-assisted distal gastrectomy. Surg Laparosc Endosc Percutan Tech 2011, 21:155-161.

20. Yamada H, Kojima K, Inokuchi M, Kawano T, Sugihara K: Effect of obesity on technical feasibility and postoperative outcomes of laparoscopy-assisted distal gastrectomy-comparison with open distal gastrectomy. J Gastrointest Surg 2008, 12:997-1004

21. Kim KH, Kim MC, Jung GJ, Kim HH: The impact of obesity on LADG for early gastric cancer. Gastric Cancer 2006, 9:303-307.

22. Cheng $Q$, Zhang B, Zhang Y, Jiang X, Zhang B, Yi B, Luo X, Wu M: Predictive factors for complications after pancreaticoduodenectomy. J Surg Res 2007, 139:22-29.

23. Pessaux P, van den Broek MA, Wu T, Olde Damink SW, Piardi T, Dejong $\mathrm{CH}$ Ntourakis D, van Dam RM: Identification and validation of risk factors for postoperative infectious complications following hepatectomy. J Gastrointest Surg 2013, 17:1907-1916.

24. McAlister FA, Bertsch K, Man J, Bradley J, Jacka M: Incidence of and risk factors for pulmonary complications after nonthoracic surgery. Am $J$ Respir Crit Care Med 2005, 171:514-517.

25. Inokuchi M, Kojima K, Kato K, Sugita H, Sugihara K: Risk factors for postoperative pulmonary complications after gastrectomy for gastric cancer. Surg Infect 2014, 15:314-321.

26. Charlson M, Szatrowski TP, Peterson J, Gold J: Validation of a combined comorbidity index. J Clin Epidemiol 1994, 47:1245-1251.

27. Park HA, Park SH, Cho SI, Jang YJ, Kim JH, Park SS, Mok YJ, Kim CS: Impact of age and comorbidity on the short-term surgical outcome after laparoscopy-assisted distal gastrectomy for adenocarcinoma. Am Surg 2013, 79:40-48.

\section{doi:10.1186/1471-2482-14-97}

Cite this article as: Inokuchi et al: Impact of comorbidities on postoperative complications in patients undergoing laparoscopy-assisted gastrectomy for gastric cancer. BMC Surgery 2014 14:97. 